\title{
Derivation of Ohm's law from the kinetic equations
}

\author{
Juhi Jang*and Nader Masmoudi ${ }^{\dagger}$
}

July 17, 2018

\begin{abstract}
The goal of this article is to give a formal derivation of Ohm's law of Magnetohydrodynamics (MHD) starting from the Vlasov-Maxwell-Boltzmann system. The derivation is based on various physical scalings and the moment methods when the Knudsen number goes to zero. We also give a derivation of the so-called Hall effect as well as other limit models such as the Navier-Stokes-Maxwell system. Our results include both the compressible and incompressible MHD models.
\end{abstract}

2000 AMS Subject Classification. 35Q20, 35Q61, 35Q83, 76W05. Keywords. Ohm's law, VlasovMaxwell-Boltzmann system, Magnetohydrodynamics, Hall effect, hydrodynamic limit

\section{Introduction}

There are different models to describe the state of a plasma depending on several parameters such as the Debye length, the plasma frequency, the collision frequencies between the different species. One of the goals of this project is give a relation between the several models used, namely how to derive rigorously the simpler models from the more complete ones and find the regimes where these approximations are valid. Formal derivation of these models can be found in Plasma Physics text books (see for instance Bellan [9], Boyd and Sanderson [12], Dendy [17] and the paper [10] etc.)

Indeed, since the plasma consists of a very large number of interacting particles, it is appropriate to adopt a statistical approach. In the kinetic description, it is only necessary to evolve the distribution function $f_{\alpha}(t, x, v)$ for each species in the system. Vlasov equation is used in this case with the Lorentz force term and collision terms. It is coupled with the Maxwell equations for the electromagnetic fields.

If collisions are important, then each species is in a local equilibrium and the plasma is treated as a fluid. More precisely it is treated as a mixture of two or more interacting fluids. This is the two-fluid model or the so-called Euler-Maxwell system.

Another level of approximation consists in treating the plasma as a single fluid by using the fact that the mass of the electrons is much smaller than the mass of the ions or by using that collisions yield that all particles will evolve (at leading order) with the same macroscopic velocity. This yields the Hall-MHD (Hall magneto-hydrodynamic model). Then, one can derive MHD models if the Hall effect is negligible.

*Department of Mathematics, University of California Riverside. Email: juhijang@math.ucr.edu Supported in part by NSF Grant DMS-0908007

${ }^{\dagger}$ Courant Institute, New York University. Email: masmoudi@cims.nyu.edu Supported in part by NSF Grant DMS-0703145 
The question on how the kinetic theory and the fluid dynamics are related is very interesting both physically and mathematically and it goes back to the founding work of Maxwell [40] and Boltzmann [11]. Moreover, the purpose of the Hilbert's sixth problem [30] is to seek a unified theory of the gas dynamics including various levels of descriptions from a mathematical point of view. There has been a lot of important progress on the hydrodynamic limits from the Boltzmann equation over the years; so far there are essentially three different mathematical approaches. The first is based on spectral analysis of the semi-group generated by the linearized Boltzmann equation; see [7, 33, 41. The second is based on Hilbert or Chapman-Enskog expansions [13, 16, 24]; see more recent work in [26, 28, 29, 32]. The third approach, initiated by Bardos-Golse-Levermore [4, 5], is working in the framework of DiPerna-Lions' renormalized solutions [18, to justify global weak solutions of incompressible flows (Navier-Stokes, Stokes, and Euler), and (compressible) acoustic system; see [5, 6, 19, 22, 34, 35, 36, 38, 42] (see also [2 for the non-cut-off case).

However, there are only limited results on hydrodynamic limits for charged particles, which satisfy the Vlasov-Maxwell-Boltzmann (VMB) system, even at the formal level due to the complexity of the system and its underlying muti-scale feature. One rigorous result is given in [31] where a diffusive expansion to VMB system was studied with one particular scaling in the incompressible regime in the framework of classical solutions [25], and as a by-product, new fluid equations interacting with the electric field, where the magnetic effect appears only at a higher order, were derived. See also [8, 27, 39] for hydrodynamic limits from the Boltzmann equations in the presence of an interacting field, but without a magnetic field.

In this article, we are interested in the derivation of MHD type equations, which describe the motion of electrically conducting media in the presence of a magnetic field, from the VMB system by introducing various scalings. As a result, we can also identify the corresponding Ohm's law of the MHD equations. The goal here is to provide formal expansions that show the relevant scalings. Our analysis is the first step towards a more rigorous analysis. It can be seen as the extension of the paper of Bardos-Golse-Levermore [4] to the VMB case. Some rigorous derivation will be given in a forthcoming paper.

Let us also mention that various MHD models were obtained from the (macroscopic) two-fluid Euler-Maxwell equations in [10] by taking different asymptotic limits. Here, our goal is to start from the kinetic level.

\subsection{Two species Vlasov-Maxwell-Boltzmann system}

The dynamics of charged dilute particles (e.g., electrons and ions) is described by the VlasovMaxwell-Boltzmann system:

$$
\begin{aligned}
& \partial_{t} F_{+}+v \cdot \nabla_{x} F_{+}+\frac{e_{+}}{m_{+}}(E+v \times B) \cdot \nabla_{v} F_{+}=Q\left(F_{+}, F_{+}\right)+Q\left(F_{+}, F_{-}\right), \\
& \partial_{t} F_{-}+v \cdot \nabla_{x} F_{-}-\frac{e_{-}}{m_{-}}(E+v \times B) \cdot \nabla_{v} F_{-}=Q\left(F_{-}, F_{+}\right)+Q\left(F_{-}, F_{-}\right), \\
& F_{ \pm}(0, x, v)=F_{0, \pm}(x, v)
\end{aligned}
$$

Here $F_{ \pm}(t, x, v) \geq 0$ are the spatially periodic number density functions for the ions $(+)$ and electrons (-) respectively, at time $t \geq 0$, position $x=\left(x_{1}, x_{2}, x_{3}\right) \in[-\pi, \pi]^{3}=\mathbf{T}^{3}$, velocity $v=\left(v_{1}, v_{2}, v_{3}\right) \in \mathbf{R}^{3}$, and $e_{ \pm}, m_{ \pm}$the magnitude of their charges and masses.

The collision between particles is given by the standard Boltzmann collision operator $Q\left(G_{1}, G_{2}\right)$ : Let $G_{1}(v), G_{2}(v)$ be two number density functions for two types of particles with 
masses $m_{i}$ and diameters $\sigma_{i}(i=1,2)$, then (p 83 and p 89 in [14]) $Q\left(G_{1}, G_{2}\right)(v)$ is defined as

$$
\begin{aligned}
& \frac{1}{4}\left(\sigma_{1}+\sigma_{2}\right)^{2} \int_{\mathbf{R}^{3} \times S^{2}} b(u-v, \omega)\left\{G_{1}\left(v^{\prime}\right) G_{2}\left(u^{\prime}\right)-G_{1}(v) G_{2}(u)\right\} d u d \omega \\
\equiv & Q_{\text {gain }}\left(G_{1}, G_{2}\right)-Q_{\text {loss }}\left(G_{1}, G_{2}\right) .
\end{aligned}
$$

For hard-sphere interaction, the collision kernel $b$ is given by $b(u-v, \omega)=|(u-v) \cdot w|$ (see [14]) and in this article, we assume the hard-sphere interaction, but the formal derivation will be valid for other general collision kernels. Here $\omega \in S^{2}$, and

$$
v^{\prime}=v-\frac{2 m_{2}}{m_{1}+m_{2}}[(v-u) \cdot \omega] \omega, \quad u^{\prime}=u+\frac{2 m_{1}}{m_{1}+m_{2}}[(v-u) \cdot \omega] \omega,
$$

which denote velocities after a collision of particles having velocities $v, u$ before the collision and vice versa. Notice that the elastic collision (1.3) implies the conservation of momentum $m_{1} v+m_{2} u$ and energy $\frac{1}{2} m_{1}|v|^{2}+\frac{1}{2} m_{2}|u|^{2}$ during the collision process. To clarify the collisions between two types of particles, we use the following notation:

$$
Q^{+} \equiv Q\left(F_{+}, F_{+}\right) ; Q^{ \pm} \equiv Q\left(F_{+}, F_{-}\right) ; Q^{\mp} \equiv Q\left(F_{-}, F_{+}\right) ; Q^{-} \equiv Q\left(F_{-}, F_{-}\right) .
$$

Note that $Q^{+}$and $Q^{-}$are the usual collision operators of one species.

The self-consistent, spatially periodic electromagnetic field $[E(t, x), B(t, x)]$ in (1.1) is coupled with $F(t, x, v)$ through the Maxwell system:

$$
\begin{aligned}
& \mu_{0} \epsilon_{0} \partial_{t} E-\nabla \times B=-\mu_{0} J=-\mu_{0} \int_{\mathbf{R}^{3}} v\left\{e_{+} F_{+}-e_{-} F_{-}\right\} d v \\
& \partial_{t} B+\nabla \times E=0, \quad \nabla \cdot B=0 \\
& \nabla \cdot E=\frac{1}{\epsilon_{0}} \int_{\mathbf{R}^{3}}\left\{e_{+} F_{+}-e_{-} F_{-}\right\} d v \\
& E(0, x)=E_{0}(x), \quad B(0, x)=B_{0}(x) .
\end{aligned}
$$

Here $\epsilon_{0}$ and $\mu_{0}$ are called the electric permittivity and the magnetic permeability of the plasma. And the speed of light $c$ is given by

$$
c^{2}=\frac{1}{\mu_{0} \epsilon_{0}} .
$$

It is well-known that for classical solutions to the VMB system, the following conservation laws of mass, total momentum (both kinetic and electromagnetic) and total energy (both kinetic and electromagnetic) hold:

$$
\begin{aligned}
& \frac{d}{d t} \int_{\mathbf{T}^{3} \times \mathbf{R}^{3}} m_{+} F_{+}(t)=0, \\
& \frac{d}{d t} \int_{\mathbf{T}^{3} \times \mathbf{R}^{3}} m_{-} F_{-}(t)=0, \\
& \frac{d}{d t}\left\{\int_{\mathbf{T}^{3} \times \mathbf{R}^{3}} v\left(m_{+} F_{+}(t)+m_{-} F_{-}(t)\right)+\frac{1}{4 \pi} \int_{\mathbf{T}^{3}} E(t) \times B(t)\right\}=0, \\
& \frac{d}{d t}\left\{\frac{1}{2} \int_{\mathbf{T}^{3} \times \mathbf{R}^{3}}|v|^{2}\left(m_{+} F_{+}(t)+m_{-} F_{-}(t)\right)+\frac{1}{8 \pi} \int_{\mathbf{T}^{3}}|E(t)|^{2}+|B(t)|^{2}\right\}=0 .
\end{aligned}
$$


Moreover, we also have the celebrated H-Theorem of Boltzmann

$$
\frac{d}{d t}\left\{\int_{\mathbf{T}^{3} \times \mathbf{R}^{3}}\left(F_{+}(t) \ln F_{+}(t)+F_{-}(t) \ln F_{-}(t)\right)\right\} \leq 0 .
$$

Indeed, the above conservation laws and the entropy dissipation can be derived from the following well-known properties of the collision operators $Q^{+}, Q^{ \pm}, Q^{\mp}, Q^{-}$. We refer to [1, 14, 15] for more details.

(i) Mass conservation:

$$
\int Q^{+} d v=0, \int Q^{ \pm} d v=0, \int Q^{\mp} d v=0, \int Q^{-} d v=0 .
$$

(ii) Momentum conservation:

$$
\int Q^{+} m_{+} v d v=0, \int\left(Q^{ \pm} m_{+} v+Q^{\mp} m_{-} v\right) d v=0, \int Q^{-} m_{-} v d v=0 .
$$

(iii) Energy conservation:

$$
\int Q^{+} m_{+}|v|^{2} d v=0, \int\left(Q^{ \pm} m_{+}|v|^{2}+Q^{\mp} m_{-}|v|^{2}\right) d v=0, \int Q^{-} m_{-}|v|^{2} d v=0 .
$$

(iv) Entropy inequalities:

$$
\begin{array}{r}
\int Q^{+}\left(F_{+}, F_{+}\right) \ln F_{+} d v \leq 0, \int Q^{-}\left(F_{-}, F_{-}\right) \ln F_{-} d v \leq 0, \\
\int\left(Q^{ \pm}\left(F_{+}, F_{-}\right) \ln F_{+}+Q^{\mp}\left(F_{-}, F_{+}\right) \ln F_{-}\right) d v \leq 0 .
\end{array}
$$

As a consequence of the entropy inequalities, we can find distribution functions (two Maxwellians) which cancel the collision operators.

(v) Local Thermodynamical Equilibria:

$$
M_{+}(t, x, v)=n_{+}\left(\frac{m_{+}}{2 \pi T}\right)^{3 / 2} e^{-m_{+}|v-\mathfrak{u}|^{2} / 2 T}, M_{-}(t, x, v)=n_{-}\left(\frac{m_{-}}{2 \pi T}\right)^{3 / 2} e^{-m_{-}|v-\mathfrak{u}|^{2} / 2 T}
$$

so that

$$
Q^{+}\left(M_{+}, M_{+}\right)+Q^{ \pm}\left(M_{+}, M_{-}\right)=0 \text { and } Q^{\mp}\left(M_{-}, M_{+}\right)+Q^{-}\left(M_{-}, M_{-}\right)=0
$$

where $n_{+}$and $n_{-}$are the ion and electron density and $\mathfrak{u}$ and $T$ are the common mean velocity and temperature and they may depend on $t$ and $x$. When the macroscopic variables do not depend on $t$ and $x$, we call them global Maxwellians. When $\mathfrak{u}=0$, we define

$$
\mu_{+}(v)=n_{+}\left(\frac{m_{+}}{2 \pi T_{0}}\right)^{3 / 2} e^{-m_{+}|v|^{2} / 2 T_{0}}, \quad \mu_{-}(v)=n_{-}\left(\frac{m_{-}}{2 \pi T_{0}}\right)^{3 / 2} e^{-m_{-}|v|^{2} / 2 T_{0}} .
$$

Note that when $e_{+} n_{+}=e_{-} n_{-}$, the global Maxwellians $\mu_{+}(v)$ and $\mu_{-}(v)$ together with $E=B=0$ define a stationary solutions to the VMB system, while the local Maxwellians are not necessarily solutions.

We now introduce the linearized collision operators over the velocity space for $Q^{+}+Q^{ \pm}$ 
and $Q^{\mp}+Q^{-}$around $M_{+}$and $M_{-}$in the following vector form:

$$
\begin{aligned}
& -\mathfrak{L}\left(\begin{array}{c}
f_{+} \\
f_{-}
\end{array}\right) \equiv \\
& \left(\begin{array}{l}
M_{+}^{-1}\left\{Q^{+}\left(M_{+} f_{+}, M_{+}\right)+Q^{+}\left(M_{+}, M_{+} f_{+}\right)+Q^{ \pm}\left(M_{+} f_{+}, M_{-}\right)+Q^{ \pm}\left(M_{+}, M_{-} f_{-}\right)\right\} \\
M_{-}^{-1}\left\{Q^{\mp}\left(M_{-} f_{-}, M_{+}\right)+Q^{\mp}\left(M_{-}, M_{+} f_{+}\right)+Q^{-}\left(M_{-} f_{-}, M_{-}\right)+Q^{-}\left(M_{-}, M_{-} f_{-}\right)\right\}
\end{array}\right) .
\end{aligned}
$$

We define the following inner product in $v$ :

$$
\left\langle\left(\begin{array}{l}
f_{+} \\
f_{-}
\end{array}\right),\left(\begin{array}{l}
g_{+} \\
g_{-}
\end{array}\right)\right\rangle_{M}=\int_{\mathbf{R}^{3}}\left(M_{+} f_{+} g_{+}+M_{-} f_{-} g_{-}\right) d v
$$

and denote by $L_{M}^{2}$ the associated Hilbert space. We will use $\langle\cdot, \cdot\rangle$ to denote the usual $L^{2}$ inner product without the weight. We summarize the properties of $\mathfrak{L}$ in the following lemma. The proof can be found in [1] (pp 635-638).

Lemma 1.1. We assume the hard-sphere interaction for the collision kernel.

1. $\mathfrak{L}$ is the sum of a diagonal operator $f \rightarrow \nu f$

$$
\nu f=\left(\begin{array}{l}
\nu_{+}(v) f_{+} \\
\nu_{-}(v) f_{-}
\end{array}\right) \text {with } \nu_{ \pm}(|v|) \sim 1+|v|
$$

and a compact operator $\mathfrak{K}$. The domain of $\mathfrak{L}$ is given by

$$
D(\mathfrak{L})=\left\{f:\left\|(1+|v|)^{\frac{1}{2}} f\right\|_{L_{M}^{2}}<\infty\right\} .
$$

2. $\mathfrak{L}$ is self-adjoint in $L_{M}^{2}$ :

$$
\left\langle\mathfrak{L}\left(\begin{array}{c}
f_{+} \\
f_{-}
\end{array}\right),\left(\begin{array}{c}
g_{+} \\
g_{-}
\end{array}\right)\right\rangle_{M}=\left\langle\left(\begin{array}{c}
f_{+} \\
f_{-}
\end{array}\right), \mathfrak{L}\left(\begin{array}{c}
g_{+} \\
g_{-}
\end{array}\right)\right\rangle_{M}
$$

$\mathfrak{L}$ is non-negative.

3. The kernel of $\mathfrak{L}$ is a six-dimensional linear space:

$$
\text { ker } \mathfrak{L}=\operatorname{Span}\left\{\left(\begin{array}{l}
1 \\
0
\end{array}\right),\left(\begin{array}{l}
0 \\
1
\end{array}\right),\left(\begin{array}{l}
m_{+} v_{i} \\
m_{-} v_{i}
\end{array}\right),\left(\begin{array}{l}
m_{+}|v|^{2} \\
m_{-}|v|^{2}
\end{array}\right)\right\} .
$$

4. Any function $f \in D(\mathfrak{L})$ can be written as $f=q_{f}+w_{f}$ with $q_{f} \in$ ker $\mathfrak{L}$ and $w_{f} \in(\text { ker } \mathfrak{L})^{\perp}$ and we have $\langle\mathfrak{L} f, f\rangle_{M} \geq \delta_{0}\left\|(1+|v|)^{\frac{1}{2}} w_{f}\right\|^{2}$.

Note that this Fredholm operator $\mathfrak{L}$ can be inverted after checking that the inhomogeneity is perpendicular to its six-dimensional null space

$$
\left\{\left(\begin{array}{l}
1 \\
0
\end{array}\right),\left(\begin{array}{l}
0 \\
1
\end{array}\right),\left(\begin{array}{l}
m_{+} v_{i} \\
m_{-} v_{i}
\end{array}\right),\left(\begin{array}{l}
m_{+}|v|^{2} \\
m_{-}|v|^{2}
\end{array}\right)\right\} \equiv\left\{\phi_{0}, \ldots, \phi_{5}\right\} .
$$

\section{$1.2 \quad$ F-G formulation}

In the MHD model, the medium is considered as a single fluid, in other words, one neglects the difference in motion of the electrons, various kinds of ions, and neutral particles. To 
derive the MHD equations from the VMB system (1.1), it is convenient to consider the total mass density and the total charge density for $F^{+}$and $F^{-}$:

$$
F \equiv m_{+} F_{+}+m_{-} F_{-} \text {and } G \equiv e_{+} F_{+}-e_{-} F_{-} .
$$

Then the VMB system (1.1) can be rewritten as

$$
\begin{gathered}
\partial_{t} F+v \cdot \nabla_{x} F+(E+v \times B) \cdot \nabla_{v} G=m_{+}\left(Q^{+}+Q^{ \pm}\right)+m_{-}\left(Q^{\mp}+Q^{-}\right), \\
\partial_{t} G+v \cdot \nabla_{x} G+(E+v \times B) \cdot \nabla_{v}\left\{\frac{e_{+} e_{-}}{m_{+} m_{-}} F+\frac{e_{+} m_{-}-e_{-} m_{+}}{m_{+} m_{-}} G\right\} \\
=e_{+}\left(Q^{+}+Q^{ \pm}\right)-e_{-}\left(Q^{\mp}+Q^{-}\right),
\end{gathered}
$$

and

$$
\begin{array}{r}
\mu_{0} \epsilon_{0} \partial_{t} E-\nabla \times B=-\mu_{0} \int_{\mathbf{R}^{3}} v G d v, \partial_{t} B+\nabla \times E=0, \\
\nabla \cdot E=\frac{1}{\epsilon_{0}} \int_{\mathbf{R}^{3}} G d v, \quad \nabla \cdot B=0 .
\end{array}
$$

Notice that the Maxwell's equations are coupled with only $G$. We will use both $F^{+}, F^{-}$ formulation (1.1) and $F, G$ formulation (1.9) throughout the article. On one hand, we remark that when $m_{+}=m_{-}(=m)$, the collision operators are of one-species and thus the right-handsides of (1.9) can be significantly simplified:

$$
\begin{aligned}
\bullet & m_{+}\left(Q^{+}+Q^{ \pm}\right)+m_{-}\left(Q^{\mp}+Q^{-}\right) \\
& =m\left\{Q\left(F_{+}, F_{+}\right)+Q\left(F_{+}, F_{-}\right)+Q\left(F_{-}, F_{+}\right)+Q\left(F_{-}, F_{-}\right)\right\} \\
& =m Q\left(F_{+}+F_{-}, F_{+}+F_{-}\right) \\
& =\frac{1}{m} Q(F, F) \\
\bullet & e_{+}\left(Q^{+}+Q^{ \pm}\right)-e_{-}\left(Q^{\mp}+Q^{-}\right) \\
\quad= & e_{+}\left\{Q\left(F_{+}, F_{+}\right)+Q\left(F_{+}, F_{-}\right)\right\}-e_{-}\left\{Q\left(F_{-}, F_{+}\right)+Q\left(F_{-}, F_{-}\right)\right\} \\
& =Q\left(e_{+} F_{+}-e_{-} F_{-}, F_{+}+F_{-}\right) \\
& =\frac{1}{m} Q(G, F)
\end{aligned}
$$

In this case, the linearized operator (1.7) is orthogonally decomposed: the linearized operator of $Q(F, F)$ has the five-dimensional kernel $\left\{1, v,|v|^{2}\right\}$ and the linearized operator of $Q(G, F)$ has the one-dimensional kernel $\{1\}$, for instance see [8, 31] for the use of these linearized operators. This special setting will be frequently used. On the other hand, we remark that when $e_{+} m_{-}=e_{-} m_{+}$, then the term $\frac{e_{+} m_{-}-e_{-} m_{+}}{m_{+} m_{-}} G$ cancels in (1.9). 


\section{Near local thermodynamical equilibria}

By using the collision invariants of the Boltzmann collision operators, if $F, G, E, B$ satisfy the VMB system (1.9) and (1.10), we formally deduce the following local conservation laws:

$$
\begin{aligned}
& \text { - } \partial_{t}\left(\int F d v\right)+\nabla_{x} \cdot\left(\int v F d v\right)=0 \\
& \text { - } \partial_{t}\left(\int v F d v\right)+\nabla_{x} \cdot\left(\int v \otimes v F d v\right)=\left(\int G d v\right) E+\left(\int v G d v\right) \times B \\
& \text { - } \partial_{t}\left(\int \frac{|v|^{2}}{2} F d v\right)+\nabla_{x} \cdot\left(\int v \frac{|v|^{2}}{2} F d v\right)=E \cdot\left(\int v G d v\right) \\
& \text { - } \partial_{t}\left(\int G d v\right)+\nabla_{x} \cdot\left(\int v G d v\right)=0
\end{aligned}
$$

which describe the conservation of the total mass, momentum, and energy and the conservation of the charge density. Of course, the equations are coupled with (1.10). However, the integrals inside $\nabla_{x}$. such as $\int v \otimes v F d v, \int v \frac{|v|^{2}}{2} F d v, \int v G d v$ are not functions of the other macroscopic quantities and they depend on the entire distributions $F$ and $G$ unless the appropriate forms for $F$ and $G$ are assumed. Thus, in general, the moment method does not give a closed form of a finite set of equations and this is often referred as the closure problem. In order to close the system of balance laws (2.1), we look for the solutions very close to local thermodynamical equilibria $F_{+} \sim M_{+}$and $F_{-} \sim M_{-}$such that in such asymptotic regime, the above integrals can be expressed in terms of the given macroscopic variables. This approximation can be realized by rescaling the system, namely by comparing the size of the system and the time scale of interest with the typical space and time scales of collisions. The task is not all trivial because the physical regimes are much richer than the pure Boltzmann equation and one needs to deal with multi-scales encoded in the VMB system.

In what follows, we introduce a few different hyperbolic scalings to the VMB system and study their hydrodynamic limits formally. Also, we discuss the Hall effect of the MHD equations. We want to point out that we did not try to get all possible scalings. In particular, one can easily derive simplified models to those we have here.

\section{$2.11 \frac{1}{2}-$ fluid Euler-Maxwell system with the common velocity and tem- perature}

We start with the simplest possible hyperbolic scaling to (1.1): Let $\tilde{t}=\varepsilon t, \tilde{x}=\varepsilon x, \tilde{F}_{ \pm}=\frac{1}{\varepsilon^{2}} F_{ \pm}$, $\tilde{b}=\varepsilon^{2} b, \tilde{E}=\frac{1}{\varepsilon} E$ and $\tilde{B}=\frac{1}{\varepsilon} B$. Dropping , we obtain the following rescaled VMB system:

$$
\begin{array}{r}
\partial_{t} F_{+}^{\varepsilon}+v \cdot \nabla_{x} F_{+}^{\varepsilon}+\frac{e_{+}}{m_{+}}\left(E^{\varepsilon}+v \times B^{\varepsilon}\right) \cdot \nabla_{v} F_{+}^{\varepsilon}=\frac{1}{\varepsilon}\left\{Q^{+}\left(F_{+}^{\varepsilon}, F_{+}^{\varepsilon}\right)+Q^{ \pm}\left(F_{+}^{\varepsilon}, F_{-}^{\varepsilon}\right)\right\}, \\
\partial_{t} F_{-}^{\varepsilon}+v \cdot \nabla_{x} F_{-}^{\varepsilon}-\frac{e_{-}}{m_{-}}\left(E^{\varepsilon}+v \times B^{\varepsilon}\right) \cdot \nabla_{v} F_{-}^{\varepsilon}=\frac{1}{\varepsilon}\left\{Q^{\mp}\left(F_{-}^{\varepsilon}, F_{+}^{\varepsilon}\right)+Q^{-}\left(F_{-}^{\varepsilon}, F_{-}^{\varepsilon}\right)\right\}, \\
\mu_{0} \epsilon_{0} \partial_{t} E^{\varepsilon}-\nabla \times B^{\varepsilon}=-\mu_{0} \int_{\mathbf{R}^{3}} v\left\{e_{+} F_{+}^{\varepsilon}-e_{-} F_{-}^{\varepsilon}\right\} d v, \partial_{t} B^{\varepsilon}+\nabla \times E^{\varepsilon}=0, \\
\nabla \cdot E^{\varepsilon}=\frac{1}{\epsilon_{0}} \int_{\mathbf{R}^{3}}\left\{e_{+} F_{+}^{\varepsilon}-e_{-} F_{-}^{\varepsilon}\right\} d v, \nabla \cdot B^{\varepsilon}=0,
\end{array}
$$

where $\varepsilon>0$ is a Knudsen number. 
Suppose $F_{ \pm}^{\varepsilon} \rightarrow F_{ \pm},\left[E^{\varepsilon}, B^{\varepsilon}\right] \rightarrow[E, B]$ as $\varepsilon \rightarrow 0$. Then from the first two equations in (2.2), we see that

$$
Q^{+}\left(F^{+}, F^{+}\right)+Q^{ \pm}\left(F^{+}, F^{-}\right)=0 ; Q^{\mp}\left(F^{-}, F^{+}\right)+Q^{-}\left(F^{-}, F^{-}\right)=0
$$

from which we deduce that

$$
F^{+}=M_{+} \text {and } F^{-}=M_{-}
$$

local Maxwellians given in (1.5). There are six fluid variables $n_{+}, n_{-}, \mathfrak{u}$ and $T$ to be determined: the dynamics of $n_{+}, n_{-}, \mathfrak{u}$ and $T$ can be derived by taking the moments of the first two equations. The system of local conservation laws

$$
\left\langle\left(\begin{array}{l}
\partial_{t} F_{+}^{\varepsilon}+v \cdot \nabla_{x} F_{+}^{\varepsilon}+\frac{e_{+}}{m_{+}}\left(E^{\varepsilon}+v \times B^{\varepsilon}\right) \cdot \nabla_{v} F_{+}^{\varepsilon} \\
\partial_{t} F_{-}^{\varepsilon}+v \cdot \nabla_{x} F_{-}^{\varepsilon}-\frac{e_{-}}{m_{-}}\left(E^{\varepsilon}+v \times B^{\varepsilon}\right) \cdot \nabla_{v} F_{-}^{\varepsilon}
\end{array}\right), \phi_{i}\right\rangle=0, i=0, \ldots, 5
$$

is, in general, not closed. If each moment converges, we can pass to the limit of $\varepsilon \rightarrow 0$. To write the equations for $n_{+}, n_{-}, \mathfrak{u}$ and $T$, we first compute the first few moments of local Maxwellians $M_{ \pm}$:

$$
\begin{aligned}
& \int M_{ \pm} d v=n_{ \pm}, \quad \int v M_{ \pm} d v=n_{ \pm} \mathfrak{u}, \quad \int|v|^{2} M_{ \pm} d v=n_{ \pm}|\mathfrak{u}|^{2}+3 \frac{n_{ \pm} T}{m_{ \pm}} \\
& \int v \otimes v M_{ \pm} d v=n_{ \pm} \mathfrak{u} \otimes \mathfrak{u}+\frac{n_{ \pm} T}{m_{ \pm}} \delta_{j}^{i}, \quad \int|v|^{2} v M_{ \pm} d v=n_{ \pm}|\mathfrak{u}|^{2} \mathfrak{u}+5 \frac{n_{ \pm} T}{m_{ \pm}} \mathfrak{u}
\end{aligned}
$$

Thus from (2.3) where $F_{ \pm}^{\varepsilon}$ are placed by $F_{ \pm}$

- $\partial_{t} n_{+}+\nabla_{x} \cdot\left(n_{+} \mathfrak{u}\right)=0$

- $\partial_{t} n_{-}+\nabla_{x} \cdot\left(n_{-} \mathfrak{u}\right)=0$

- $\left.\partial_{t}\left[\left(m_{+} n_{+}+m_{-} n_{-}\right) \mathfrak{u}\right]+\nabla_{x} \cdot\left[\left(m_{+} n_{+}+m_{-} n_{-}\right) \mathfrak{u} \otimes \mathfrak{u}\right]+\nabla_{x}\left[\left(n_{+}+n_{-}\right) T\right)\right]$ $=\left(n_{+} e_{+}-n_{-} e_{-}\right) E+\left(n_{+} e_{+}-n_{-} e_{-}\right) \mathfrak{u} \times B$

- $\partial_{t}\left[\frac{1}{2}\left(m_{+} n_{+}+m_{-} n_{-}\right)|\mathfrak{u}|^{2}+\frac{3}{2}\left(n_{+}+n_{-}\right) T\right]$

$$
+\nabla_{x} \cdot\left[\frac{1}{2}\left(m_{+} n_{+}+m_{-} n_{-}\right)|\mathfrak{u}|^{2} \mathfrak{u}+\frac{5}{2}\left(n_{+}+n_{-}\right) T \mathfrak{u}\right]=E \cdot\left[\left(n_{+} e_{+}-n_{-} e_{-}\right) \mathfrak{u}\right]
$$

with

$$
\begin{array}{r}
\mu_{0} \epsilon_{0} \partial_{t} E-\nabla \times B=-\mu_{0}\left(e_{+} n_{+}-e_{-} n_{-}\right) \mathfrak{u} ; \partial_{t} B+\nabla \times E=0 ; \\
\nabla \cdot E=\frac{1}{\epsilon_{0}}\left(e_{+} n_{+}-e_{-} n_{-}\right) ; \nabla \cdot B=0 .
\end{array}
$$

By introducing the total density $\rho$ and the charge density $\sigma$ :

$$
m_{+} n_{+}+m_{-} n_{-}=: \rho \text { and } e_{+} n_{+}-e_{-} n_{-}=: \sigma
$$


the above system (2.4) and (2.5) can be equivalently written as

$$
\begin{aligned}
& -\partial_{t} \rho+\nabla_{x} \cdot(\rho \mathfrak{u})=0 \\
& \text { - } \partial_{t}(\rho \mathfrak{u})+\nabla_{x} \cdot(\rho \mathfrak{u} \otimes \mathfrak{u})+\nabla_{x}\left[\left(n_{+}+n_{-}\right) T\right]=\sigma E+\sigma \mathfrak{u} \times B \\
& \text { - } \partial_{t}\left[\frac{\rho|\mathfrak{u}|^{2}}{2}+\frac{3}{2}\left(n_{+}+n_{-}\right) T\right]+\nabla_{x} \cdot\left[\frac{\rho|\mathfrak{u}|^{2} \mathfrak{u}}{2}+\frac{5}{2}\left(n_{+}+n_{-}\right) T \mathfrak{u}\right]=\sigma E \cdot \mathfrak{u} \\
& \text { - } \partial_{t} \sigma+\nabla_{x} \cdot(\sigma \mathfrak{u})=0
\end{aligned}
$$

where

$$
\mu_{0} \epsilon_{0} \partial_{t} E-\nabla \times B=-\mu_{0} \sigma \mathfrak{u} ; \partial_{t} B+\nabla \times E=0 ; \nabla \cdot E=\frac{1}{\epsilon_{0}} \sigma ; \nabla \cdot B=0
$$

and

$$
n_{ \pm}=\frac{e_{\mp} \rho \pm m_{\mp} \sigma}{e_{-} m_{+}+e_{+} m_{-}} \text {and } n_{+}+n_{-}=\frac{\left(e_{+}+e_{-}\right) \rho+\left(m_{-}-m_{+}\right) \sigma}{e_{-} m_{+}+e_{+} m_{-}} .
$$

The first three equations in (2.6) represent the balance laws of total mass, total momentum, and total energy and the last equation is the conservation of the charge density. In this regime, the current density $J$ is given by $\sigma \mathfrak{u}$. Hence, by letting

$$
p=\left(n_{+}+n_{-}\right) T \text { (ideal gas law), }
$$

the solvability condition (2.3) yields Euler-Maxwell type equations (2.6) and (2.7) having the same mean velocity and temperature fields which are therefore not exactly of two-fluid. From (2.8), note that for a special case of $m_{+}=m_{-}$, the pressure is induced by the total density but in general, it depends on the charge density as well.

We have proved the following theorem.

Theorem 2.1. Let $F_{ \pm}^{\varepsilon},\left[E^{\varepsilon}, B^{\varepsilon}\right]$ be the classical solutions to (2.2). Assume that $F_{ \pm}^{\varepsilon} \rightarrow F_{ \pm}$, $\left[E^{\varepsilon}, B^{\varepsilon}\right] \rightarrow[E, B]$ strongly as $\varepsilon \rightarrow 0$. Then the limit $F_{ \pm}$is a Maxwellian given by (1.5) and moreover, $n_{ \pm}, \mathfrak{u}, T, E, B$ solve the $1 \frac{1}{2}$-fluid Euler-Maxwell system (2.4) and (2.5).

For a rigorous proof of this result, we can argue as in Caflisch [13. Indeed, the derivation given above can also be used to provide a Hilbert expansion as in [13. This is then used to provide an error estimate between the solution to the $1 \frac{1}{2}$-fluid Euler-Maxwell system and the solutions to the VMB system (2.2) in the limit when $\varepsilon$ goes to zero.

\subsection{Compressible resistive MHD}

We have seen that it is convenient to write the equations in terms of the total density and the charge density in the previous section. In this subsection, we start with the $F-G$ formulation introduced in Section 1.2. We set $m_{+}=m_{-}=e_{+}=e_{-}=1$ for simplicity of the presentation. Then by using (1.11), the VMB system (1.9) reads as follows:

$$
\begin{aligned}
& \partial_{t} F+v \cdot \nabla_{x} F+(E+v \times B) \cdot \nabla_{v} G=Q(F, F), \\
& \partial_{t} G+v \cdot \nabla_{x} G+(E+v \times B) \cdot \nabla_{v} F=Q(G, F) .
\end{aligned}
$$

In order to see the ideal MHD type equations with Ohm's law, we consider the quasi-neutral regime so that $G \sim 0$. We next introduce another scaling to (2.9) as follows: Let $\tilde{t}=\varepsilon t$, $\tilde{x}=\varepsilon x, \tilde{E}=\frac{1}{\sqrt{\varepsilon}} E, \tilde{B}=\frac{1}{\sqrt{\varepsilon}} B, \tilde{G}=\frac{1}{\varepsilon^{3 / 2}} G, \tilde{F}=\frac{1}{\varepsilon} F$, and $\tilde{b}=\varepsilon b$ where $E=O(\sqrt{\varepsilon})$, 
$B=O(\sqrt{\varepsilon}), G=O\left(\varepsilon^{3 / 2}\right)$ and $F=O(\varepsilon)$. Dropping , we obtain the following rescaled VMB system:

$$
\begin{aligned}
& \partial_{t} F^{\varepsilon}+v \cdot \nabla_{x} F^{\varepsilon}+\left(E^{\varepsilon}+v \times B^{\varepsilon}\right) \cdot \nabla_{v} G^{\varepsilon}=\frac{1}{\varepsilon} Q\left(F^{\varepsilon}, F^{\varepsilon}\right), \\
& \partial_{t} G^{\varepsilon}+v \cdot \nabla_{x} G^{\varepsilon}+\left(\frac{E^{\varepsilon}}{\varepsilon}+\frac{v \times B^{\varepsilon}}{\varepsilon}\right) \cdot \nabla_{v} F^{\varepsilon}=\frac{1}{\varepsilon} Q\left(G^{\varepsilon}, F^{\varepsilon}\right),
\end{aligned}
$$

coupled with the Maxwell's equations

$$
\begin{array}{r}
\mu_{0} \epsilon_{0} \partial_{t} E^{\varepsilon}-\nabla \times B^{\varepsilon}=-\mu_{0} \int v G^{\varepsilon} d v, \quad \partial_{t} B^{\varepsilon}+\nabla \times E^{\varepsilon}=0, \\
\nabla \cdot E^{\varepsilon}=\frac{1}{\epsilon_{0}} \int G^{\varepsilon} d v, \quad \nabla \cdot B^{\varepsilon}=0 .
\end{array}
$$

Suppose that there exists a classical solution $\left(F^{\varepsilon}, G^{\varepsilon}, E^{\varepsilon}, B^{\varepsilon}\right)$ to (2.10) and (2.11) and that there exist $F, G, E, B$ so that $F^{\varepsilon} \rightarrow F, G^{\varepsilon} \rightarrow G, E^{\varepsilon} \rightarrow E, B^{\varepsilon} \rightarrow B$ as $\varepsilon \rightarrow 0$. Then from (2.10), as $\varepsilon \rightarrow 0$ we deduce that

$$
Q(F, F)=0 \Longrightarrow F=M=\frac{\rho}{(2 \pi T)^{3 / 2}} e^{-|v-\mathfrak{u}|^{2} / 2 T}
$$

also

$$
(E+v \times B) \cdot \nabla_{v} F=Q(G, F) .
$$

This case, it is already different from the previous ones in that $G$ is not determined by a Maxwellian. Of course here $G$ is a higher order fluctuation. Define

$$
\mathcal{L} g=-\frac{1}{M} Q(M g, M)
$$

where $M$ is the Maxwellian given in (2.12). Then from Lemma 1.1 we deduce that ker $\mathcal{L}=$ $\operatorname{span}\{1\}$. The solvability condition for $G$ is automatically satisfied and thus by inverting the linearized operator $\mathcal{L}$ in (2.13) we derive that

$$
G=-M\left[\mathcal{L}^{-1}\left\{\frac{1}{M}(E+v \times B) \cdot \nabla_{v} M\right\}+\psi_{0}\right], \text { where } \mathcal{L} \psi_{0}=0 .
$$

Let us define the first two moments of $G$ by $\sigma$ (the charge density) and $J$ (the current):

$$
\sigma:=\int G d v \text { and } J:=\int v G d v
$$

Then from (2.14) we can write the current $J$ as

$$
J=\int v G d v=\langle v, G\rangle=-\left\langle v, M \mathcal{L}^{-1}\left\{\frac{1}{M}(E+v \times B) \cdot \nabla_{v} M\right\}\right\rangle
$$

and since

$$
\frac{1}{M}(E+v \times B) \cdot \nabla_{v} M=-(E+v \times B) \cdot \frac{(v-\mathfrak{u})}{T}=-\frac{1}{T}(E+\mathfrak{u} \times B) \cdot(v-\mathfrak{u})
$$


and by using the fact that $\mathcal{L}^{-1}$ acts only in $v$, we obtain

$$
\begin{aligned}
J & =\left\langle v, M \mathcal{L}^{-1}\left\{\frac{1}{T}(E+\mathfrak{u} \times B) \cdot(v-\mathfrak{u})\right\}\right\rangle=\left\langle v M, \frac{1}{T}(E+\mathfrak{u} \times B) \cdot \mathcal{L}^{-1}(v-\mathfrak{u})\right\rangle \\
& =\left\langle(v-\mathfrak{u}) M, \frac{1}{T}(E+\mathfrak{u} \times B) \cdot \mathcal{L}^{-1}(v-\mathfrak{u})\right\rangle .
\end{aligned}
$$

Since $\int\left(v^{i}-\mathfrak{u}^{i}\right) \mathcal{L}^{-1}\left(v^{j}-\mathfrak{u}^{j}\right) M d v=0$ for $i \neq j$, the last expression for $J$ can be reduced to the following:

$$
J=\frac{1}{\eta}(E+\mathfrak{u} \times B)
$$

where

$$
\frac{1}{\eta}=\frac{1}{3 T} \int(v-\mathfrak{u}) \cdot \mathcal{L}^{-1}(v-\mathfrak{u}) M d v>0 .
$$

Here, the number 3 represents the space dimension 3. The relation (2.16) is called the Ohm's law. $\eta$ is called resistivity and $1 / \eta$ is the conductivity. Next we take the moments of $F$ equation in (2.10), then by collision invariants of $Q$, we get

$$
\left\langle\partial_{t} F^{\varepsilon}+v \cdot \nabla_{x} F^{\varepsilon}+\left(E^{\varepsilon}+v \times B^{\varepsilon}\right) \cdot \nabla_{v} G^{\varepsilon},\left\{1, v, \frac{|v|^{2}}{2}\right\}\right\rangle=0
$$

and

$$
\begin{array}{r}
\mu_{0} \epsilon_{0} \partial_{t} E^{\varepsilon}-\nabla \times B^{\varepsilon}=-\mu_{0} \int v G^{\varepsilon} d v, \quad \partial_{t} B^{\varepsilon}+\nabla \times E^{\varepsilon}=0, \\
\nabla \cdot E^{\varepsilon}=\frac{1}{\epsilon_{0}} \int G^{\varepsilon} d v, \quad \nabla \cdot B^{\varepsilon}=0 .
\end{array}
$$

We pass to the limit and by using the fact that $F$ is a Maxwellain for which we can explicitly compute the moments and by using the notation introduced in (2.15), we obtain the following resistive MHD type equations for $\rho, \mathfrak{u}, T, \sigma, J, E, B$ :

- $\partial_{t} \rho+\nabla_{x} \cdot(\rho \mathfrak{u})=0$

- $\partial_{t}(\rho \mathfrak{u})+\nabla_{x} \cdot(\rho \mathfrak{u} \otimes \mathfrak{u})+\nabla_{x}(\rho T)=\sigma E+J \times B$

- $\partial_{t}\left(\frac{\rho|\mathfrak{u}|^{2}}{2}+\frac{3}{2} \rho T\right)+\nabla_{x} \cdot\left(\frac{\rho|\mathfrak{u}|^{2} \mathfrak{u}}{2}+\frac{5}{2} \rho T \mathfrak{u}\right)=E \cdot J$

- $\partial_{t} \sigma+\nabla_{x} \cdot J=0$

- $J=\frac{1}{\eta}(E+\mathfrak{u} \times B)$

- $\mu_{0} \epsilon_{0} \partial_{t} E-\nabla \times B=-\mu_{0} J, \partial_{t} B+\nabla \times E=0, \quad \nabla \cdot B=0, \nabla \cdot E=\frac{\sigma}{\epsilon_{0}}$.

We have proved the following theorem.

Theorem 2.2. Let $\left[F^{\varepsilon}, G^{\varepsilon}\right],\left[E^{\varepsilon}, B^{\varepsilon}\right]$ be the classical solutions to (2.10) and (2.11). Assume that $\left[F^{\varepsilon}, G^{\varepsilon}\right] \rightarrow[F, G],\left[E^{\varepsilon}, B^{\varepsilon}\right] \rightarrow[E, B]$ strongly as $\varepsilon \rightarrow 0$. Then the limit $F$ is a Maxwellian given by (2.12) and moreover, $\rho, \mathfrak{u}, T, \sigma, J, E, B$ solve the electromagneto-hydrodynamic system (2.19).

The electromagnetic field in the system (2.19) is still governed by the full Maxwell's 
equations; if we consider the regime where the electric permittivity $\epsilon_{0}$ is sufficiently small, namely if we consider the quasi-neutral limit, then we would get a new system in which the magnetic field becomes dominant. We may set $\epsilon_{0} \sim \varepsilon$ by fixing $\mu_{0}$ in the Maxwell's equations (2.11) to obtain different approximations to the dynamics of the electromagnetic field. Namely, if we consider

$$
\mu_{0} \varepsilon \partial_{t} E^{\varepsilon}-\nabla \times B^{\varepsilon}=-\mu_{0} \int v G^{\varepsilon} d v \text { and } \nabla \cdot E^{\varepsilon}=\frac{1}{\varepsilon} \int G^{\varepsilon} d v,
$$

following the same procedure, we would get $\nabla \times B=\mu_{0} J$ and $\sigma=0$. And (2.19) reduces to

- $\partial_{t} \rho+\nabla_{x} \cdot(\rho \mathfrak{u})=0$

- $\partial_{t}(\rho \mathfrak{u})+\nabla_{x} \cdot(\rho \mathfrak{u} \otimes \mathfrak{u})+\nabla_{x}(\rho T)=J \times B$

- $\partial_{t}\left(\frac{\rho|\mathfrak{u}|^{2}}{2}+\frac{3}{2} \rho T\right)+\nabla_{x} \cdot\left(\frac{\rho|\mathfrak{u}|^{2} \mathfrak{u}}{2}+\frac{5}{2} \rho T \mathfrak{u}\right)=E \cdot J$

- $J=\frac{1}{\eta}(E+\mathfrak{u} \times B)$

- $\nabla \times B=\mu_{0} J, \quad \partial_{t} B+\nabla \times E=0, \quad \nabla \cdot B=0$

which are the well-known compressible resistive MHD equations.

Theorem 2.3. Let $\left[F^{\varepsilon}, G^{\varepsilon}\right],\left[E^{\varepsilon}, B^{\varepsilon}\right]$ be the classical solutions to (2.10) and (2.11) where $\epsilon_{0}$ is taken to be $\varepsilon$. Assume that $\left[F^{\varepsilon}, G^{\varepsilon}\right] \rightarrow[F, G],\left[E^{\varepsilon}, B^{\varepsilon}\right] \rightarrow[E, B]$ strongly as $\varepsilon \rightarrow 0$. Then the limit $F$ is a Maxwellian given by (2.12) and moreover, $\rho, \mathfrak{u}, T, J$ and $E, B$ solve the compressible resistive MHD equations (2.20).

We remark that the ideal MHD equations are valid when the resistivity $\eta$ is negligible.

Also, we point out that the simplification of setting the physical constants to be one allows us to treat the collision operators decoupled as in (2.9). The General case, in particular when $m_{+} \neq m_{-}$, can be treated similarly. In such case, we have to start with (1.1) or (1.9) and need to treat the collision operators as matrix form as in (1.7) and use that it has a six dimensional kernel. We do not detail this here.

\subsection{When $e_{-} m_{+} \neq e_{+} m_{-}$and Hall effect}

The Hall effect is known as two-fluid effect. In our setting, the Hall effect can be captured when $e_{-} m_{+} \neq e_{+} m_{-}$at the higher order. For instance, we take the same scaling as done in (2.10) to (1.9),

$$
\begin{gathered}
\partial_{t} F^{\varepsilon}+v \cdot \nabla_{x} F^{\varepsilon}+\left(E^{\varepsilon}+v \times B^{\varepsilon}\right) \cdot \nabla_{v} G^{\varepsilon}=\frac{1}{\varepsilon}\left\{m_{+}\left(Q^{+}+Q^{ \pm}\right)+m_{-}\left(Q^{\mp}+Q^{-}\right)\right\}, \\
\partial_{t} G^{\varepsilon}+v \cdot \nabla_{x} G^{\varepsilon}+\left(\frac{E^{\varepsilon}}{\varepsilon}+\frac{v \times B^{\varepsilon}}{\varepsilon}\right) \cdot \nabla_{v}\{\frac{e_{+} e_{-}}{m_{+} m_{-}} F^{\varepsilon}+\varepsilon^{\frac{1}{2}} \underbrace{\frac{e_{+} m_{-}-e_{-} m_{+}}{m_{+} m_{-}} G^{\varepsilon}}_{(*)}\} \\
=\frac{1}{\varepsilon}\left[e_{+}\left(Q^{+}+Q^{ \pm}\right)-e_{-}\left(Q^{\mp}+Q^{-}\right)\right] .
\end{gathered}
$$

The main difference is the presence of the term $(*)$, which will affect the Ohm's law.

Following the same spirit of the Chapman-Enskog expansion, if we also keep the $\mathrm{O}(\sqrt{\varepsilon})$ terms in the second equation of (2.21), instead of (2.16), we would get the following Ohm's 
law

$$
\eta J=\frac{e_{+} e_{-}}{m_{+} m_{-}}(E+\mathfrak{u} \times B)+\frac{\varepsilon^{\frac{1}{2}}}{\rho}\left(\frac{e_{+} m_{-}-e_{-} m_{+}}{m_{+} m_{-}}\right)(\sigma E+J \times B)
$$

where $J \times B$ is often referred to as the Hall effect term. We remark that this Hall effect is not seen by the moment method that we have used for the previous theorems but rather this can be captured by the higher order Chapman-Enskog type expansions. It would be interesting to justify this higher order effect in a rigorous framework.

Remark 2.4. We have chosen our scalings so far based on the appropriate time, space and forcing with fixed masses $m_{+}$and $m_{-}$. When $m_{-} \ll m_{+}$and $\frac{m_{-}}{m_{+}}$is comparable to Knudsen number, one can also parametrize $\frac{m_{-}}{m_{+}}$. By doing so, for example, we can get the rescaled system (2.10) or (2.21) without rescaling forcing terms. In such regime, one may want to study the appropriate collision operators as suggested in [15].

\section{The incompressible regime}

As we have seen in the previous section, the derivation of hydrodynamic equations from the Boltzmann equation is closely related to finding approximate solutions of the Boltzmann equation, because the fluid variables are defined and change in space and time scales that are very large when measured in the units of the mean free path and mean free time between collisions. In the present section, we'd like to discuss the longer time scaling. We will mainly work on the simplified case where the constants are taken to be unity and present general cases at the end when we discuss the Hall effect.

\subsection{Incompressible Navier-Stokes-Fourier-Maxwell equations}

Recall the system (2.9) with the Maxwell's equations:

$$
\begin{aligned}
& \partial_{t} F+v \cdot \nabla_{x} F+(E+v \times B) \cdot \nabla_{v} G=Q(F, F), \\
& \partial_{t} G+v \cdot \nabla_{x} G+(E+v \times B) \cdot \nabla_{v} F=Q(G, F), \\
& \mu_{0} \epsilon_{0} \partial_{t} E-\nabla \times B=-\mu_{0} \int_{\mathbb{R}^{3}} v G d v, \nabla \cdot B=0, \\
& \partial_{t} B+\nabla \times E=0, \quad \nabla \cdot E=\frac{1}{\epsilon_{0}} \int_{\mathbb{R}^{3}} G d v,
\end{aligned}
$$

where $F=F_{+}+F_{-}$is the density for the whole particles and $G=F_{+}-F_{-}$represents the disparity between two species (the charge density).

We will discuss how to obtain the incompressible Navier-Stokes-Fourier-Maxwell system. To keep the hyperbolic structure of Maxwell's equations, we take $\mu_{0}$ and $\epsilon_{0}$ as $O(1)$ and let $\tilde{t}=\varepsilon t, \tilde{x}=\varepsilon x, \tilde{v}=\varepsilon v$ (longer time scale), $\tilde{E}=\varepsilon E, \tilde{B}=\varepsilon B$, and $\tilde{F}=\varepsilon F$. Then the system 
(3.1) can be rescaled as follows: by dropping ,

$$
\begin{aligned}
& \varepsilon \partial_{t} F^{\varepsilon}+v \cdot \nabla_{x} F^{\varepsilon}+\left(\varepsilon E^{\varepsilon}+v \times B^{\varepsilon}\right) \cdot \nabla_{v} G^{\varepsilon}=\frac{1}{\varepsilon} Q\left(F^{\varepsilon}, F^{\varepsilon}\right), \\
& \varepsilon \partial_{t} G^{\varepsilon}+v \cdot \nabla_{x} G^{\varepsilon}+\left(\frac{E^{\varepsilon}}{\varepsilon}+\frac{v \times B^{\varepsilon}}{\varepsilon^{2}}\right) \cdot \nabla_{v} F^{\varepsilon}=\frac{1}{\varepsilon} Q\left(G^{\varepsilon}, F^{\varepsilon}\right), \\
& \mu_{0} \epsilon_{0} \partial_{t} E^{\varepsilon}-\nabla \times B^{\varepsilon}=-\mu_{0} \int_{\mathbb{R}^{3}} v G^{\varepsilon} d v, \nabla \cdot B^{\varepsilon}=0, \\
& \partial_{t} B^{\varepsilon}+\nabla \times E^{\varepsilon}=0, \quad \nabla \cdot E^{\varepsilon}=\frac{1}{\epsilon_{0}} \int_{\mathbb{R}^{3}} G^{\varepsilon} d v .
\end{aligned}
$$

We now consider the following perturbation of $F^{\varepsilon}, G^{\varepsilon}, E^{\varepsilon}, B^{\varepsilon}$ around $[\mu, 0,0,0]$ :

$$
F^{\varepsilon}=\mu\left(1+\varepsilon f_{1}^{\varepsilon}\right), G^{\varepsilon}=\varepsilon \mu g_{1}^{\varepsilon}, E^{\varepsilon}=\varepsilon E_{1}^{\varepsilon}, B^{\varepsilon}=\varepsilon B_{1}^{\varepsilon}
$$

where $\mu$ is a global Maxwellian normalized as

$$
\frac{1}{(2 \pi)^{3 / 2}} e^{-|v|^{2} / 2}
$$

Suppose that there exist the classical solutions $f_{1}^{\varepsilon}, g_{1}^{\varepsilon}, E_{1}^{\varepsilon}, B_{1}^{\varepsilon}$ to (3.2) and further assume that as $\varepsilon \rightarrow 0, f_{1}^{\varepsilon} \rightarrow f, g_{1}^{\varepsilon} \rightarrow g, E_{1}^{\varepsilon} \rightarrow E, B_{1}^{\varepsilon} \rightarrow B$. Under this convergence assumption, we will derive the dynamics of the limiting variables $f, g, E, B$. We first rewrite the system (3.2) in terms of the perturbed variables:

$$
\begin{aligned}
& \varepsilon \partial_{t} f_{1}^{\varepsilon}+v \cdot \nabla_{x} f_{1}^{\varepsilon}+\varepsilon\left(\varepsilon E_{1}^{\varepsilon}+v \times B_{1}^{\varepsilon}\right) \cdot \frac{1}{\mu} \nabla_{v}\left(\mu g_{1}^{\varepsilon}\right) \\
& =\frac{1}{\varepsilon \mu}\left\{Q\left(\mu f_{1}^{\varepsilon}, \mu\right)+Q\left(\mu, \mu f_{1}^{\varepsilon}\right)+\varepsilon Q\left(\mu f_{1}^{\varepsilon}, \mu f_{1}^{\varepsilon}\right)\right\} \\
& \varepsilon \partial_{t} g_{1}^{\varepsilon}+v \cdot \nabla_{x} g_{1}^{\varepsilon}+\left(\frac{E^{\varepsilon}}{\varepsilon}+\frac{v \times B^{\varepsilon}}{\varepsilon^{2}}\right) \cdot \frac{1}{\mu} \nabla_{v}\left(\mu+\varepsilon \mu f_{1}^{\varepsilon}\right) \\
& =\frac{1}{\varepsilon \mu}\left\{Q\left(\mu g_{1}^{\varepsilon}, \mu\right)+\varepsilon Q\left(\mu g_{1}^{\varepsilon}, \mu f_{1}^{\varepsilon}\right)\right\}, \\
& \mu_{0} \epsilon_{0} \partial_{t} E_{1}^{\varepsilon}-\nabla \times B_{1}^{\varepsilon}=-\mu_{0} \int_{\mathbb{R}^{3}} v \mu g_{1}^{\varepsilon} d v, \nabla \cdot B_{1}^{\varepsilon}=0, \\
& \partial_{t} B_{1}^{\varepsilon}+\nabla \times E_{1}^{\varepsilon}=0, \quad \nabla \cdot E_{1}^{\varepsilon}=\frac{1}{\epsilon_{0}} \int_{\mathbb{R}^{3}} \mu g_{1}^{\varepsilon} d v .
\end{aligned}
$$

We recall the linearized collision operators $L$ and $\mathcal{L}$ (see for instance [8, 31]):

$$
L f=-\frac{1}{\mu}\{Q(\mu f, \mu)+Q(\mu, \mu f)\} \text { and } \mathcal{L} g=-\frac{1}{\mu} Q(\mu g, \mu) .
$$

Note that $[L, \mathcal{L}]$ is equivalent to the linearized collision operator introduced in (1.7). We also recall that $\operatorname{ker} L=\operatorname{span}\left\{1, v,|v|^{2}\right\}$ and $\operatorname{ker} \mathcal{L}=\operatorname{span}\{1\}$.

By taking $\varepsilon \rightarrow 0$ in the first two equations in (3.3) and using the convergence assumption, we first obtain

$$
0=-L f
$$


and

$$
-E \cdot v+\frac{1}{\mu}(v \times B) \cdot \nabla_{v}(\mu f)=-\mathcal{L} g .
$$

Since $f$ is in the kernel of $L$ by (3.4), $f$ can be written as

$$
f=\rho+v \cdot u+\left(\frac{|v|^{2}}{2}-\frac{3}{2}\right) \theta .
$$

We also introduce

$$
\sigma:=\int g \mu d v \text { and } J:=\int g v \mu d v .
$$

From (3.5), we can write the current $J$ as

$$
J=\int g v \mu d v=\int \mathcal{L}^{-1}\left(E \cdot v-\frac{1}{\mu}(v \times B) \cdot \nabla_{v}(\mu f)\right) v \mu d v .
$$

Since

$$
-\frac{1}{\mu}(v \times B) \cdot \nabla_{v}(\mu f)=-v \times B \cdot \nabla_{v} f=-v \times B \cdot u=u \times B \cdot v
$$

and by using the fact that $\mathcal{L}^{-1}$ acts only in $v$, we obtain

$$
J=\int\left(\mathcal{L}^{-1} v\right) \cdot(E+u \times B) v \mu d v .
$$

Since $\int\left(\mathcal{L}^{-1} v^{i}\right) v^{j} \mu d v=0$ for $i \neq j$, we can derive the following Ohm's law

$$
J=\frac{1}{\eta}(E+u \times B)
$$

where

$$
\frac{1}{\eta}=\frac{1}{3} \int\left(\mathcal{L}^{-1} v\right) \cdot v \mu d v
$$

The derivation of the dynamics of $\rho, u, \theta$ is very similar to the derivation of the incompressible Navier-Stokes-Fourier system (for instance see [4]) except the presence of $E, B$ and $g$. The local conservation laws of mass and momentum of the first equation in (3.3) are written as

$$
\begin{aligned}
& \varepsilon\left\langle\partial_{t} f_{1}^{\varepsilon}, \mu\right\rangle+\nabla_{x} \cdot\left\langle f_{1}^{\varepsilon}, v \mu\right\rangle=0 \\
& \varepsilon\left\langle\partial_{t} f_{1}^{\varepsilon}, v \mu\right\rangle+\nabla_{x} \cdot\left\langle f_{1}^{\varepsilon}, v \otimes v \mu\right\rangle+\varepsilon^{2}\left\langle E_{1}^{\varepsilon} \cdot \nabla_{v}\left(\mu g_{1}^{\varepsilon}\right), v\right\rangle+\varepsilon\left\langle\left(v \times B_{1}^{\varepsilon}\right) \cdot \nabla_{v}\left(\mu g_{1}^{\varepsilon}\right), v\right\rangle=0
\end{aligned}
$$

where we have used $\int\left(v \times B_{1}\right) \cdot \nabla_{v}\left(\mu g_{1}\right) d v=0$. By letting $\varepsilon$ go to zero, we see that

$$
\nabla_{x} \cdot\langle f, v \mu\rangle=0 \text { and } \nabla_{x} \cdot\langle f, v \otimes v \mu\rangle=0
$$

which yield the divergence-free condition and the Boussinesq relation

$$
\nabla_{x} \cdot u=0 \text { and } \nabla_{x}(\rho+\theta)=0 .
$$


The limiting momentum equation is obtained from

$$
\underbrace{\left\langle\partial_{t} f_{1}^{\varepsilon}, v \mu\right\rangle}_{(a)}+\underbrace{\frac{1}{\varepsilon} \nabla_{x} \cdot\left\langle f_{1}^{\varepsilon}, v \otimes v \mu\right\rangle}_{(b)}+\underbrace{\varepsilon\left\langle E_{1}^{\varepsilon} \cdot \nabla_{v}\left(\mu g_{1}^{\varepsilon}\right), v\right\rangle}_{(c)}+\underbrace{\left\langle\left(v \times B_{1}^{\varepsilon}\right) \cdot \nabla_{v}\left(\mu g_{1}^{\varepsilon}\right), v\right\rangle}_{(d)}=0 .
$$

The first two terms, treated in [4, give rise to $(a) \rightarrow \partial_{t} u$ and $(b) \rightarrow u \cdot \nabla_{x} u+\nabla_{x} p-\nu \Delta u$ and here the viscosity $\nu$ depends on the collision operator $L$. See the section 4 in [4] for more detail for the treatment of $(b)$. Note that $(c) \rightarrow 0$. And for $(d)$, we see that

$$
(d) \rightarrow \int v(v \times B) \cdot \nabla_{v}(\mu g) d v=-J \times B .
$$

Hence, we obtain the following momentum equation

$$
\partial_{t} u+u \cdot \nabla u+\nabla p=\nu \Delta u+J \times B .
$$

Similarly from the local conservation law of the energy, we can derive the equation for $\theta$. Since

$$
\int \frac{|v|^{2}}{2}\left(v \times B_{1}\right) \cdot \nabla_{v}\left(\mu g_{1}\right) d v=-\int\left(v \times B_{1}\right) \cdot v \mu g_{1} d v=0
$$

we indeed get the same Fourier equation as in the pure Boltzmann equation

$$
\partial_{t} \theta+u \cdot \nabla \theta=\kappa \Delta \theta
$$

Together with the limiting Maxwell's equations, we deduce that $\rho, u, \theta, J, E, B$ satisfy the incompressible Navier-Stokes-Fourier-Maxwell system:

- $\rho+\theta=0, \nabla \cdot u=0$

- $\partial_{t} u+u \cdot \nabla u+\nabla p=\nu \Delta u+J \times B$

- $\partial_{t} \theta+u \cdot \nabla \theta=\kappa \Delta \theta$

- $E+u \times B=\eta J$

- $\mu_{0} \epsilon_{0} \partial_{t} E-\nabla \times B=-\mu_{0} J, \partial_{t} B+\nabla \times E=0, \nabla \cdot B=0$

This system was studied in [37]. We have established the following result:

Theorem 3.1. Let $\left[f_{1}^{\varepsilon}, g_{1}^{\varepsilon}, E_{1}^{\varepsilon}, B_{1}^{\varepsilon}\right]$ be the classical solutions to (3.3). Assume that $f_{1}^{\varepsilon}, g_{1}^{\varepsilon}$, $E_{1}^{\varepsilon}, B_{1}^{\varepsilon}$ converge strongly to $f, g, E, B$ as $\varepsilon \rightarrow 0$. Then the limit $f$ has the form $f=\rho+v \cdot u+$ $\left(\frac{|v|^{2}}{2}-\frac{3}{2}\right) \theta$. Moreover, $\rho, u, \theta, J, E, B$ solve the incompressible Navier-Stokes-Fourier-Maxwell system (3.9). 


\subsection{Incompressible viscous MHD}

We now discuss the viscous MHD limit. We consider the same scaling as in (3.2) and in addition, we take $\epsilon_{0}=\varepsilon$ so that the electric permittivity is sufficiently small:

$$
\begin{aligned}
& \varepsilon \partial_{t} F^{\varepsilon}+v \cdot \nabla_{x} F^{\varepsilon}+\left(\varepsilon E^{\varepsilon}+v \times B^{\varepsilon}\right) \cdot \nabla_{v} G^{\varepsilon}=\frac{1}{\varepsilon} Q\left(F^{\varepsilon}, F^{\varepsilon}\right), \\
& \varepsilon \partial_{t} G^{\varepsilon}+v \cdot \nabla_{x} G^{\varepsilon}+\left(\frac{E^{\varepsilon}}{\varepsilon}+\frac{v \times B^{\varepsilon}}{\varepsilon^{2}}\right) \cdot \nabla_{v} F^{\varepsilon}=\frac{1}{\varepsilon} Q\left(G^{\varepsilon}, F^{\varepsilon}\right), \\
& \mu_{0} \varepsilon \partial_{t} E^{\varepsilon}-\nabla \times B^{\varepsilon}=-\mu_{0} \int_{\mathbb{R}^{3}} v G^{\varepsilon} d v, \nabla \cdot B^{\varepsilon}=0, \\
& \partial_{t} B^{\varepsilon}+\nabla \times E^{\varepsilon}=0, \quad \nabla \cdot E^{\varepsilon}=\frac{1}{\varepsilon} \int_{\mathbb{R}^{3}} G^{\varepsilon} d v .
\end{aligned}
$$

As before, we consider the following perturbation of $F^{\varepsilon}, G^{\varepsilon}, E^{\varepsilon}, B^{\varepsilon}$ around $[\mu, 0,0,0]$ :

$$
F^{\varepsilon}=\mu\left(1+\varepsilon f_{1}^{\varepsilon}\right), G^{\varepsilon}=\varepsilon \mu g_{1}^{\varepsilon}, E^{\varepsilon}=\varepsilon E_{1}^{\varepsilon}, B^{\varepsilon}=\varepsilon B_{1}^{\varepsilon}
$$

and we suppose that there exist the classical solutions $f_{1}^{\varepsilon}, g_{1}^{\varepsilon}, E_{1}^{\varepsilon}, B_{1}^{\varepsilon}$ to (3.2) and further assume that as $\varepsilon \rightarrow 0, f_{1}^{\varepsilon} \rightarrow f, g_{1}^{\varepsilon} \rightarrow g, E_{1}^{\varepsilon} \rightarrow E, B_{1}^{\varepsilon} \rightarrow B$. Following the same procedure as in the previous section, we can derive the equations for $\rho, u, \theta, J, E, B$. The only difference from the previous system (3.9) lies in the Maxwell's equations. The last equation in (3.10) forces $\sigma=0$ when $\varepsilon \rightarrow 0$ and the third equation in (3.10) yields the Amperes law $\nabla \times B=\mu_{0} J$. We obtain the following theorem:

Theorem 3.2. Let $F^{\varepsilon}=\mu\left(1+\varepsilon f_{1}^{\varepsilon}\right), G^{\varepsilon}=\varepsilon \mu g_{1}^{\varepsilon}, E^{\varepsilon}=\varepsilon E_{1}^{\varepsilon}, B^{\varepsilon}=\varepsilon B_{1}^{\varepsilon}$ be the classical solutions to (3.10). Assume that $f_{1}^{\varepsilon}, g_{1}^{\varepsilon}, E_{1}^{\varepsilon}, B_{1}^{\varepsilon}$ converge strongly to $f, g, E, B$ as $\varepsilon \rightarrow 0$. Then the limit $f$ has the form $f=\rho+v \cdot u+\left(\frac{|v|^{2}}{2}-\frac{3}{2}\right) \theta$. Moreover, $\rho, u, \theta, J, E, B$ solve the following incompressible Navier-Stokes-Fourier-MHD equations:

- $\rho+\theta=0, \nabla \cdot u=0$

- $\partial_{t} u+u \cdot \nabla u+\nabla p=\nu \Delta u+J \times B$

- $\partial_{t} \theta+u \cdot \nabla \theta=\kappa \Delta \theta$

- $E+u \times B=\eta J$

- $\nabla \times B=\mu_{0} J, \partial_{t} B+\nabla \times E=0, \nabla \cdot B=0$

\subsection{Incompressible inviscid MHD}

To see how the incompressible inviscid MHD system can be derived, we introduce the following scaling to (3.1) by letting $\tilde{t}=\varepsilon^{3} t, \tilde{x}=\varepsilon^{2} x, \tilde{E}=\frac{1}{\varepsilon^{3 / 2}} E, \tilde{B}=\frac{1}{\sqrt{\varepsilon}} B, \tilde{G}=\frac{1}{\varepsilon^{3 / 2}} G$. By dropping ,

$$
\begin{aligned}
& \varepsilon \partial_{t} F^{\varepsilon}+v \cdot \nabla_{x} F^{\varepsilon}+\left(\varepsilon E^{\varepsilon}+v \times B^{\varepsilon}\right) \cdot \nabla_{v} G^{\varepsilon}=\frac{1}{\varepsilon^{2}} Q\left(F^{\varepsilon}, F^{\varepsilon}\right), \\
& \varepsilon \partial_{t} G^{\varepsilon}+v \cdot \nabla_{x} G^{\varepsilon}+\frac{1}{\varepsilon^{2}}\left(E^{\varepsilon}+\frac{v \times B^{\varepsilon}}{\varepsilon}\right) \cdot \nabla_{v} F^{\varepsilon}=\frac{1}{\varepsilon^{2}} Q\left(G^{\varepsilon}, F^{\varepsilon}\right), \\
& \mu_{0} \epsilon_{0} \varepsilon \partial_{t} E^{\varepsilon}-\frac{1}{\varepsilon^{2}} \nabla \times B^{\varepsilon}=-\frac{\mu_{0}}{\varepsilon^{2}} \int_{\mathbb{R}^{3}} v G^{\varepsilon} d v, \nabla \cdot B^{\varepsilon}=0, \\
& \partial_{t} B^{\varepsilon}+\nabla \times E^{\varepsilon}=0, \quad \nabla \cdot E^{\varepsilon}=\frac{1}{\epsilon_{0} \varepsilon^{2}} \int_{\mathbb{R}^{3}} G^{\varepsilon} d v .
\end{aligned}
$$


We consider the following perturbation of $F^{\varepsilon}, G^{\varepsilon}, E^{\varepsilon}, B^{\varepsilon}$ around $[\mu, 0,0,0]$ :

$$
F^{\varepsilon}=\mu\left(1+\varepsilon f_{1}^{\varepsilon}\right), G^{\varepsilon}=\varepsilon \mu g_{1}^{\varepsilon}, E^{\varepsilon}=\varepsilon E_{1}^{\varepsilon}, B^{\varepsilon}=\varepsilon B_{1}^{\varepsilon} .
$$

We plug this expansion into (3.12) to obtain the similar expression to (3.3):

$$
\begin{gathered}
\varepsilon \partial_{t} f_{1}^{\varepsilon}+v \cdot \nabla_{x} f_{1}^{\varepsilon}+\varepsilon\left(\varepsilon E_{1}^{\varepsilon}+v \times B_{1}^{\varepsilon}\right) \cdot \frac{1}{\mu} \nabla_{v}\left(\mu g_{1}^{\varepsilon}\right) \\
=\frac{1}{\varepsilon^{2} \mu}\left\{Q\left(\mu f_{1}^{\varepsilon}, \mu\right)+Q\left(\mu, \mu f_{1}^{\varepsilon}\right)+\varepsilon Q\left(\mu f_{1}^{\varepsilon}, \mu f_{1}^{\varepsilon}\right)\right\} \\
\varepsilon \partial_{t} g_{1}^{\varepsilon}+v \cdot \nabla_{x} g_{1}^{\varepsilon}+\left(\frac{E^{\varepsilon}}{\varepsilon^{2}}+\frac{v \times B^{\varepsilon}}{\varepsilon^{3}}\right) \cdot \frac{1}{\mu} \nabla_{v}\left(\mu+\varepsilon \mu f_{1}^{\varepsilon}\right) \\
=\frac{1}{\varepsilon^{2} \mu}\left\{Q\left(\mu g_{1}^{\varepsilon}, \mu\right)+\varepsilon Q\left(\mu g_{1}^{\varepsilon}, \mu f_{1}^{\varepsilon}\right)\right\} \\
\mu_{0} \epsilon_{0} \varepsilon \partial_{t} E_{1}^{\varepsilon}-\frac{1}{\varepsilon^{2}} \nabla \times B_{1}^{\varepsilon}=-\frac{\mu_{0}}{\varepsilon^{2}} \int_{\mathbb{R}^{3}} v \mu g_{1}^{\varepsilon} d v, \nabla \cdot B_{1}^{\varepsilon}=0, \\
\partial_{t} B_{1}^{\varepsilon}+\nabla \times E_{1}^{\varepsilon}=0, \quad \nabla \cdot E_{1}^{\varepsilon}=\frac{1}{\epsilon_{0} \varepsilon^{2}} \int_{\mathbb{R}^{3}} \mu g_{1}^{\varepsilon} d v .
\end{gathered}
$$

As before, under the convergence assumption of the solutions, we deduce that $f=\rho+v$. $u+\left(\frac{|v|^{2}}{2}-\frac{3}{2}\right) \theta$. and also we can derive the same Ohm's law (3.6). We can follow the same procedure as in the previous sections to derive the equations for $\rho, u, \theta, J, E, B$. The difference is the faster relaxation $\frac{1}{\varepsilon^{2}}$ than the previous cases and the diffusion for fluid variables doesn't appear at the first order. We can obtain the following theorem.

Theorem 3.3. Suppose that there exist the classical solutions $f_{1}^{\varepsilon}, g_{1}^{\varepsilon}, E_{1}^{\varepsilon}, B_{1}^{\varepsilon}$ to (3.13) and further assume that as $\varepsilon \rightarrow 0, f_{1}^{\varepsilon} \rightarrow f, g_{1}^{\varepsilon} \rightarrow g, E_{1}^{\varepsilon} \rightarrow E, B_{1}^{\varepsilon} \rightarrow B$. Then $f=\rho+v \cdot u+$ $\left(\frac{|v|^{2}}{2}-\frac{3}{2}\right) \theta$. and the limiting equations can be recorded as follows:

$$
\begin{aligned}
& \text { - } \rho+\theta=0, \nabla \cdot u=0 \\
& \text { - } \partial_{t} u+u \cdot \nabla u+\nabla p=J \times B \\
& \text { - } \partial_{t} \theta+u \cdot \nabla \theta=0 \\
& \text { - } E+u \times B=\eta J \\
& \text { - } \nabla \times B=\mu_{0} J, \partial_{t} B+\nabla \times E=0, \nabla \cdot B=0
\end{aligned}
$$

which we refer to the incompressible inviscid (Euler) resistive MHD equations.

We remark that the incompressible, inviscid, ideal MHD equations are valid when the resistivity $\eta$ is negligible.

\subsection{Different masses and Hall effect}

We now look at the case when $m_{+} \neq m_{-}$. Moreover, we consider the quasi-neutral regime where $n_{+}=n_{-}=1$, we assume the same charges $e_{+}=e_{-}=1$, and that we choose $T_{0}=1$ such that

$$
\int \mu_{+} d v=\int \mu_{-} d v=1
$$


where $\mu_{+}$and $\mu_{-}$are given in (1.6). We also assume that

$$
\int\left(m_{+} \mu_{+}+m_{-} \mu_{-}\right) d v=m_{+}+m_{-}=1
$$

We take the same parabolic scaling for $t, x, E, B$ as done in (3.10) to (1.1) except for the scaling of $G$ :

$$
\begin{aligned}
& \varepsilon \partial_{t} F_{+}^{\varepsilon}+v \cdot \nabla_{x} F_{+}^{\varepsilon}+\frac{1}{m_{+}}\left(E^{\varepsilon}+v \times \frac{B^{\varepsilon}}{\varepsilon}\right) \cdot \nabla_{v} F_{+}^{\varepsilon}=\frac{1}{\varepsilon} Q\left(F_{+}^{\varepsilon}, F_{+}^{\varepsilon}\right)+\frac{1}{\varepsilon} Q\left(F_{+}^{\varepsilon}, F_{-}^{\varepsilon}\right), \\
& \varepsilon \partial_{t} F_{-}^{\varepsilon}+v \cdot \nabla_{x} F_{-}^{\varepsilon}-\frac{1}{m_{-}}\left(E^{\varepsilon}+v \times \frac{B^{\varepsilon}}{\varepsilon}\right) \cdot \nabla_{v} F_{-}^{\varepsilon}=\frac{1}{\varepsilon} Q\left(F_{-}^{\varepsilon}, F_{+}^{\varepsilon}\right)+\frac{1}{\varepsilon} Q\left(F_{-}^{\varepsilon}, F_{-}^{\varepsilon}\right) .
\end{aligned}
$$

We then consider the following expansion of the solutions near the global Maxwellians $\mu_{+}$ and $\mu_{-}$:

$$
F_{+}^{\varepsilon}=\mu_{+}\left(1+\varepsilon f_{+}^{\varepsilon}\right), \quad F_{-}^{\varepsilon}=\mu_{-}\left(1+\varepsilon f_{-}^{\varepsilon}\right), \quad E^{\varepsilon}=\varepsilon E_{1}^{\varepsilon}, \quad B^{\varepsilon}=\varepsilon B_{1}^{\varepsilon} .
$$

The system (3.15) can be rewritten as

$$
\begin{aligned}
\varepsilon \partial_{t} f_{+}^{\varepsilon} & +v \cdot \nabla_{x} f_{+}^{\varepsilon}+\frac{1}{m_{+} \mu_{+}}\left(E_{1}^{\varepsilon}+v \times \frac{B_{1}^{\varepsilon}}{\varepsilon}\right) \cdot \nabla_{v}\left(\mu_{+}+\varepsilon \mu_{+} f_{+}^{\varepsilon}\right) \\
& =-\frac{1}{\varepsilon} \mathfrak{L}^{1}\left(\begin{array}{c}
f_{+}^{\varepsilon} \\
f_{-}^{\varepsilon}
\end{array}\right)+\frac{1}{\mu_{+}}\left\{Q\left(\mu_{+} f_{+}^{\varepsilon}, \mu_{+} f_{+}^{\varepsilon}\right)+Q\left(\mu_{+} f_{+}^{\varepsilon}, \mu_{-} f_{-}^{\varepsilon}\right)\right\}, \\
\varepsilon \partial_{t} f_{-}^{\varepsilon} & +v \cdot \nabla_{x} f_{-}^{\varepsilon}-\frac{1}{m_{-} \mu_{-}}\left(E_{1}^{\varepsilon}+v \times \frac{B_{1}^{\varepsilon}}{\varepsilon}\right) \cdot \nabla_{v}\left(\mu_{-}+\varepsilon \mu_{-} f_{-}^{\varepsilon}\right) \\
& =-\frac{1}{\varepsilon} \mathfrak{L}^{2}\left(\begin{array}{c}
f_{+}^{\varepsilon} \\
f_{-}^{\varepsilon}
\end{array}\right)+\frac{1}{\mu_{-}}\left\{Q\left(\mu_{-} f_{-}^{\varepsilon}, \mu_{+} f_{+}^{\varepsilon}\right)+Q\left(\mu_{-} f_{-}^{\varepsilon}, \mu_{-} f_{-}^{\varepsilon}\right)\right\},
\end{aligned}
$$

where we have used $\mathfrak{L}^{1}$ and $\mathfrak{L}^{2}$ to denote each component of the linearized collision operator $\mathfrak{L}$ given in (1.7). By taking $\varepsilon \rightarrow 0$, we see that

$$
\mathfrak{L}\left(\begin{array}{l}
f^{+} \\
f^{-}
\end{array}\right)=0
$$

namely

$$
\left(\begin{array}{l}
f^{+}(t, x, v) \\
f^{-}(t, x, v)
\end{array}\right)=n^{+}(t, x)\left(\begin{array}{l}
1 \\
0
\end{array}\right)+n^{-}(t, x)\left(\begin{array}{l}
0 \\
1
\end{array}\right)+u(t, x) \cdot\left(\begin{array}{l}
m_{+} v \\
m_{-} v
\end{array}\right)+\theta(t, x)\left(\begin{array}{c}
\frac{m_{+}|v|^{2}}{2}-\frac{3}{2} \\
\frac{m_{-}|v|^{2}}{2}-\frac{3}{2}
\end{array}\right)
$$


where $n^{+}, n^{-}, u, \theta$ are to be determined. Then the local conservation laws of mass and momentum yield

$$
\begin{aligned}
& \varepsilon\left\langle\partial_{t}\left(\begin{array}{c}
f_{+}^{\varepsilon} \\
f_{-}^{\varepsilon}
\end{array}\right),\left(\begin{array}{c}
1 \\
0
\end{array}\right)\right\rangle_{M}+\nabla_{x} \cdot\left\langle\left(\begin{array}{c}
f_{+}^{\varepsilon} \\
f_{-}^{\varepsilon}
\end{array}\right),\left(\begin{array}{l}
v \\
0
\end{array}\right)\right\rangle_{M}=0, \\
& \varepsilon\left\langle\partial_{t}\left(\begin{array}{c}
f_{+}^{\varepsilon} \\
f_{-}^{\varepsilon}
\end{array}\right),\left(\begin{array}{c}
0 \\
1
\end{array}\right)\right\rangle_{M}+\nabla_{x} \cdot\left\langle\left(\begin{array}{c}
f_{+}^{\varepsilon} \\
f_{-}^{\varepsilon}
\end{array}\right),\left(\begin{array}{l}
0 \\
v
\end{array}\right)\right\rangle_{M}=0, \\
& \varepsilon\left\langle\partial_{t}\left(\begin{array}{c}
f_{+}^{\varepsilon} \\
f_{-}^{\varepsilon}
\end{array}\right),\left(\begin{array}{l}
m_{+} v \\
m_{-} v
\end{array}\right)\right\rangle_{M}+\nabla_{x} \cdot\left\langle\left(\begin{array}{c}
f_{+}^{\varepsilon} \\
f_{-}^{\varepsilon}
\end{array}\right),\left(\begin{array}{l}
m_{+} v \otimes v \\
m_{-} v \otimes v
\end{array}\right)\right\rangle_{M} \\
& \quad+\left\langle\left(\varepsilon E_{1}^{\varepsilon}+v \times B_{1}^{\varepsilon}\right) \cdot \nabla_{v}\left(\mu_{+} f_{+}^{\varepsilon}-\mu_{-} f_{-}^{\varepsilon}\right), v\right\rangle=0 .
\end{aligned}
$$

As $\varepsilon \rightarrow 0$, since $\left\langle\left(v \times B_{1}\right) \cdot \nabla_{v}\left(\mu_{+} f_{+}-\mu_{-} f_{-}\right), v\right\rangle=-\left\langle v, \mu_{+} f_{+}-\mu_{-} f_{-}\right\rangle \times B_{1}=0 \times B_{1}=0$, we deduce that

$$
\begin{array}{r}
\nabla_{x} \cdot\left\langle\left(\begin{array}{c}
f_{+} \\
f_{-}
\end{array}\right),\left(\begin{array}{l}
v \\
0
\end{array}\right)\right\rangle_{M}=0, \nabla_{x} \cdot\left\langle\left(\begin{array}{c}
f_{+} \\
f_{-}
\end{array}\right),\left(\begin{array}{l}
v \\
0
\end{array}\right)\right\rangle_{M}=0 \quad \Longrightarrow \quad \nabla_{x} \cdot u=0 \\
\nabla_{x} \cdot\left\langle\left(\begin{array}{c}
f_{+} \\
f_{-}
\end{array}\right),\left(\begin{array}{c}
m_{+} v \otimes v \\
m_{-} v \otimes v
\end{array}\right)\right\rangle_{M}=0 \quad \Longrightarrow \quad \nabla_{x}(\rho+\theta)=0
\end{array}
$$

where $\rho=n_{+}+n_{-}$. We remark that

$$
J_{0} \equiv \int v\left(\mu_{+} f_{+}-\mu_{-} f_{-}\right) d v=0
$$

By assuming that

$$
\lim _{\varepsilon \rightarrow 0} \frac{1}{\varepsilon} J^{\varepsilon}=J_{1}
$$

one can derive the MHD type equations containing $J_{1} \times B_{1}$ as before.

To discuss the Ohm's law, we write out the dynamics of the charge density $G^{\varepsilon}:=\mu_{+} f_{+}^{\varepsilon}-$ $\mu_{-} f_{-}^{\varepsilon}$ from (3.17):

$$
\begin{aligned}
\varepsilon \partial_{t} G^{\varepsilon}+v \cdot \nabla_{x} G^{\varepsilon}+\left(E_{1}^{\varepsilon}+v \times \frac{B_{1}^{\varepsilon}}{\varepsilon}\right) \cdot \nabla_{v}\left(\frac{\mu_{+}}{m_{+}}+\frac{\mu_{-}}{m_{-}}+\varepsilon\left(\frac{\mu_{+} f_{+}^{\varepsilon}}{m_{+}}+\frac{\mu_{-} f_{-}^{\varepsilon}}{m_{-}}\right)\right) \\
=-\frac{1}{\varepsilon}\left(\mu_{+} \mathfrak{L}^{1}\left(\begin{array}{c}
f_{+}^{\varepsilon} \\
f_{-}^{\varepsilon}
\end{array}\right)-\mu_{-} \mathfrak{L}^{2}\left(\begin{array}{c}
f_{+}^{\varepsilon} \\
f_{-}^{\varepsilon}
\end{array}\right)\right) \\
\quad+\left\{Q\left(\mu_{+} f_{+}^{\varepsilon}, \mu_{+} f_{+}^{\varepsilon}\right)+Q\left(\mu_{+} f_{+}^{\varepsilon}, \mu_{-} f_{-}^{\varepsilon}\right)-Q\left(\mu_{-} f_{-}^{\varepsilon}, \mu_{+} f_{+}^{\varepsilon}\right)-Q\left(\mu_{-} f_{-}^{\varepsilon}, \mu_{-} f_{-}^{\varepsilon}\right)\right\}
\end{aligned}
$$

Now projecting (3.20) onto $v$, we obtain the dynamics of $J^{\varepsilon}=\left\langle v G^{\varepsilon}\right\rangle$ :

$$
\begin{aligned}
& \varepsilon \partial_{t} J^{\varepsilon}+\nabla_{x} \cdot\left\langle v \otimes v G^{\varepsilon}\right\rangle-\left(\frac{1}{m_{+}}+\frac{1}{m_{-}}+\varepsilon\left(\frac{n_{+}^{\varepsilon}}{m_{+}}+\frac{n_{-}^{\varepsilon}}{m_{-}}\right)\right) E_{1}^{\varepsilon} \\
& -\underbrace{\left(\frac{\left\langle v, \mu_{+} f_{+}^{\varepsilon}\right\rangle}{m_{+}}+\frac{\left\langle v, \mu_{-} f_{-}^{\varepsilon}\right\rangle}{m_{-}}\right) \times B_{1}^{\varepsilon}}_{(*)}=-\frac{1}{\varepsilon} \eta J^{\varepsilon}+\left\langle v, Q\left(\mu_{+} f_{+}^{\varepsilon}, \mu_{-} f_{-}^{\varepsilon}\right)-Q\left(\mu_{-} f_{-}^{\varepsilon}, \mu_{+} f_{+}^{\varepsilon}\right)\right\rangle
\end{aligned}
$$

To derive the Ohm's law, we split the last term $(*)$ in the left-hand-side of (3.21) into two 
parts:

$$
\begin{aligned}
(*) & =\frac{\left\langle v, m_{+} \mu_{+} f_{+}^{\varepsilon}+m_{-} \mu_{-} f_{-}^{\varepsilon}\right\rangle}{m_{+} m_{-}} \times B_{1}^{\varepsilon}+\left(\frac{1}{m_{+}}-\frac{1}{m_{-}}\right)\left\langle v, \mu_{+} f_{+}^{\varepsilon}-\mu_{-} f_{-}^{\varepsilon}\right\rangle \times B_{1}^{\varepsilon} \\
& =\frac{1}{m_{+} m_{-}} u^{\varepsilon} \times B_{1}^{\varepsilon}+\underbrace{\left(\frac{1}{m_{+}}-\frac{1}{m_{-}}\right) J^{\varepsilon} \times B_{1}^{\varepsilon}}_{(* *)} .
\end{aligned}
$$

By (3.18), $(* *) \rightarrow 0$. Hence the Ohm's law for $J_{1} \equiv \lim _{\varepsilon \rightarrow 0} \frac{1}{\varepsilon} J^{\varepsilon}$ is given by

$$
\eta J_{1}=\frac{1}{m_{+} m_{-}}\left(E_{1}+u \times B_{1}\right)+\nabla_{x} P+\mathcal{C}
$$

in the limit $\varepsilon \rightarrow 0$. Here $\left[E_{1}, B_{1}\right]=\lim _{\varepsilon \rightarrow 0}\left[E_{1}^{\varepsilon}, B_{1}^{\varepsilon}\right], u=\lim _{\varepsilon \rightarrow 0}\left\langle v, m_{+} \mu_{+} f_{+}^{\varepsilon}+m_{-} \mu_{-} f_{-}^{\varepsilon}\right\rangle$, $\nabla_{x} P=-\nabla_{x} \cdot\left\langle v \otimes v G_{0}\right\rangle$ where $G_{0}=\lim _{\varepsilon \rightarrow 0} G^{\varepsilon}$, and the pressure $P$ may depend on $\frac{n^{-}}{m_{-}}-\frac{n^{+}}{m_{+}}$ and $\left(\frac{1}{m_{-}}-\frac{1}{m_{+}}\right) \theta$. Notice that we didn't have the pressure term in the previous simplified cases because we started with the strong quasi-neutral scaling where $G_{0}=0$. The last term $\mathcal{C}$ coming from the last term in (3.21) is given by $\left\langle v, Q\left(\mu_{+} f_{+}, \mu_{-} f_{-}\right)-Q\left(\mu_{-} f_{-}, \mu_{+} f_{+}\right)\right\rangle$. In fact, $f_{+}$and $f_{-}$are purely hydrodynamic described by $n^{ \pm}, u, \theta$ and hence $\mathcal{C}$ may depend on $n^{ \pm}, u, \theta$ quadratically. Notice that $\mathcal{C}$ vanishes when $m_{+}=m_{-}$.

Now we briefly discuss the Hall effect in the above setting. As in the compressible case, the Hall effect is not seen in the first order limit. The Hall effect is represented by the Hall term $J_{1} \times B_{1}$, which should be seen from $(* *)$ in (3.22). Since $\lim _{\varepsilon \rightarrow 0} J^{\varepsilon}=0$ by (3.18), $(* *)$ is 0 at the leading order. If we approximate $J^{\varepsilon}$ by $\varepsilon J_{1}$ by using (3.19), we see that $(* *)=\varepsilon\left(\frac{1}{m_{+}}-\frac{1}{m_{-}}\right) J_{1} \times B_{1}+o(\varepsilon)$. Therefore, we deduce that the Hall term $J_{1} \times B_{1}$ in (3.21) can be captured at the next order by including $O(\varepsilon)$ terms in the spirit of Chapman-Enskog type expansions. It is interesting to point out that $(* *)$ is always 0 for $m_{+}=m_{-}$and thus it will not affect the Ohm's law at all; for instance, see Section 3.1 and 3.2. This confirms that the Hall effect is a two-fluid effect. In the case of $m_{+} \neq m_{-}$, the general Ohm's law (3.21) is much more complicated in that not only the Hall effect but also other higher order effects might appear. This motivates us to study the higher order expansion which will be investigated in a separate article.

\section{Conclusion}

We would like to conclude the paper with few remarks:

1) In this paper, we tried to give a formal derivation in the spirit of [4 to MHD type models starting form the Vlasov-Maxwell-Boltzmann system. For compressible models, we were able to derive the $1 \frac{1}{2}$ Euler-Maxwell system. It seems to us that the classical two fluid Euler-Maxwell system can not be derived from the model we started from and we plan to come back to this problem in a forthcoming work

2) We were also able to derive Ohm's law and to compute the resistivity from the Boltzmann kernel. This can be compared to the formulas giving the viscosity $\nu$ and heat diffusivity $\kappa$ in [4. It is worth pointing out that in our derivation the Hall effect which yields a correction to the Ohm's law can only be seen as a higher order correction. This is similar to the compressible Navier-Stokes system which is a correction to the compressible Euler system.

3) There are few other important scaling parameters that we did not take into account in 
these derivation. One of them is the mass ratio between the electron and the ions. Usually it reflects the fact that the electrons attain their equilibrium much faster than the ions.

4) As observed by Grad [23], Ohm's law is also valid through an entirely different mechanism, the so-called gyro-oscillation. We will leave this (magnetic) gyro-effect with or without collisions for future study.

5) After most of this work was done, we learnt that D. Arsenio and L. Saint-Raymond [3] are also studying similar limits starting form the renormalized solutions of DiPerna and Lions.

Acknowledgements. The authors would like to thank the referees for helpful comments and suggestions, which have improved the presentation of the paper. Part of work was done during the authors' visit to ICERM, Brown University in the fall of 2011 and they thank the institute for the support.

\section{References}

[1] K. Aoki,C. Bardos, S. Takata: Knudsen layer for gas mixtures, J. Statist. Phys. 112 (2003), 629-655

[2] D. Arsenio. Hydrodynamic limit with long-range interactions. ARMA (to appear), 2011.

[3] D. Arsenio, L. Saint-Raymond personal communication. 2011.

[4] C. Bardos, F. Golse, D. Levermore: Fluid dynamic limits of kinetic equations. I Formal derivations, J. Statist. Phys. 63, 323-344 (1991)

[5] C. Bardos, F. Golse, D. Levermore: Fluid dynamic limits of kinetic equations. II convergence proofs for the Boltzmann equation, Comm. Pure appl. Math. 46, 667-753 (1993)

[6] C. Bardos, F. Golse, D. Levermore: The acoustic limit for the Boltzmann equation, Arch. Rational. Mech. Anal. 153, 177-204 (2000)

[7] C. Bardos, S. Ukai: The classical incompressible Navier-Stokes limit of the Boltzmann equation. Math. Models Methods Appl. Sci. 1 (1991), 235-257

[8] S. Bastea, R. Esposito, J. L. Lebowitz, R. Marra: Binary fluids with long range segregating interaction. I: Derivation of kinetic and hydrodynamic equations, J. Statist. Phys. 101, 1087-1136 (2000)

[9] P. M. Bellan. Fundamentals of plasmas physics. Cambridge University Press, Cambridge, 2006.

[10] Besse, Christophe; Degond, Pierre; Deluzet, Fabrice; Claudel, Jean; GalLiCE, Grard; Tessieras: Christian A model hierarchy for ionospheric plasma modeling. Math. Models Methods Appl. Sci. 14 (2004), no. 3, 393-415

[11] L. Boltzmann: Weitere Studien über das Wärmegleichgewicht unter Gasmolekülen. Sitzungs. Akad. Wiss. Wien 66 (1872), 275-370. English translation: Further studies on the thermal equilibrium of gas molecules. Kinetic theory, vol. 2, 88-174. Pergamon, London, 1966. 
[12] T. J. M. Boyd and J. J. Sanderson. The physics of plasmas. Cambridge University Press, Cambridge, 2003.

[13] R. Caflisch: The fluid dynamic limit of the nonlinear Boltzmann equation, Comm. Pure Appl. Math. 33 (1980), 651-666

[14] Chapman, S., Cowling, T. G., The Mathematical Theory of Non-uniform Gases. Cambridge, 1970.

[15] P. Degond: Asymptotic continuum models for plasmas and disparate mass gaseous binary mixtures, in 'Material substructures in complex bodies: from atomic level to continuum', G. Capriz, P-M. Mariano (eds), Elsevier, Amsterdam, 2007

[16] A. De Masi, R. Esposito, J.L. Lebowitz: Incompressible Navier-Stokes and Euler limits of the Boltzmann equation, Comm.Pure.Appl.Math. 42 (1989), 1189-1214

[17] R.-O. Dendy. Plasma dynamics. Oxford University Press, 1990.

[18] R. DiPerna, P.-L. Lions: On the Cauchy problem for the Boltzmann equations: global existence and weak stability, Ann. of Math. 130, 321-366 (1989)

[19] F. Golse, D. Levermore: Stokes-Fourier and Acoustic limits for the Boltzmann equation: Convergence proofs, Comm. Pure Appl. Math. 55, 336-393 (2002)

[20] F. Golse, L. Saint-Raymond: The Vlasov-Poisson system with strong magnetic field, J. Math. Pures Appl. 78, 791-817 (1999)

[21] F. Golse, L. Saint-Raymond: The Vlasov-Poisson system with strong magnetic field in quasineutral regime, Math. Models Methods Appl. Sci. 13, 661-714 (2003)

[22] F. Golse, L. Saint-Raymond: The Navier-Stokes limit of the Boltzmann equation for bounded collision kernels, Invent. Math. 155, 81-161 (2004)

[23] H. Grad: Notes on Magneto-Hydrodynamics IV: Ohm's Law, Courant Institute of Mathematical Sciences, New York University, Doc. NYO-6486-IV (1956)

[24] H. GraD: Asymptotic theory of the Boltzmann equation. II, Rarefied Gas Dynamics (Proc. 3rd Internat. Sympos., Palais de IUNESCO, Paris, 1962), Vol. I, 1963, pp. 26-59

[25] Y. Guo: The Vlasov-Maxwell-Boltzmann system near Maxwellians, Invent. Math. 153, 593-630 (2003)

[26] Y. Guo: Boltzmann diffusive limit beyond the Navier-Stokes approximation, Comm. Pure Appl. Math. 59, 626-687 (2006)

[27] Y. Guo, J. JANG: Global Hilbert expansion for the Vlasov-Poisson-Boltzmann system. Comm. Math. Phys. 299 (2010), no. 2, 469-501

[28] Y. Guo, J. Jang, N. JiAng: Local Hilbert expansion for the Boltzmann equation. Kinet. Relat. Models 2 (2009), no. 1, 20-214.

[29] Y. GuO; J. JANG; N. JiAng: Acoustic limit for the Boltzmann equation in optimal scaling. Comm. Pure Appl. Math. 63 (2010), no. 3, 33-361 
[30] D. Hilbert: Mathematical Problems, ICM Paris 1900, translated and reprinted in Bull. Amer. Soc. 37 (2000), 407-436

[31] J. Jang: Vlasov-Maxwell-Boltzmann diffusive limit. Arch. Rational Mech. Anal. 194 (2009), no. 2, 531-584

[32] J. JANG, N. JiAng: Acoustic limit of the Boltzmann equation: classical solutions. Discrete Contin. Dyn. Syst. 25 (2009), no. 3, 869-882

[33] S. Kawashima, S. Matsumura, T. Nishida: On the fluid-dynamical approximation to the Boltzmann equation at the level of the Navier-Stokes equation. Comm. Math. Phys. 70 (1979), 97-124

[34] D. Levermore, N. Masmoudi: From the Boltzamnn equation to an incompressible Navier-Stokes-Fourier system, Arch. Ration. Mech. Anal. 196 (2010), 753-809

[35] P.-L. Lions, N. Masmoudi: From Boltzmann equations to incompressible fluid mechanics equation. I, Arch. Rational. Mech. Anal. 158, 173-193 (2001)

[36] P.-L. Lions, N. Masmoudi: From Boltzmann equations to incompressible fluid mechanics equation. II, Arch. Rational. Mech. Anal. 158, 195-211 (2001)

[37] N. Masmoudi: Global well posedness for the Maxwell-Navier-Stokes system in 2D. J. Math. Pures Appl. (9) 93 (2010), no. 6, 559-571

[38] N. Masmoudi, L. Saint-Raymond: From the Boltzmann equation to Stokes-Fourier system in a bounded domain, Comm. Pure Appl. Math. 56, 1263-1293 (2003)

[39] N. Masmoudi, M.L. Tayeb: Diffusion limit of a semiconductor Boltzmann-Poisson system, SIAM J. Math. Anal. 38, 1788-1807 (2007)

[40] J. C. Maxwell: On the dynamical Theory of Gases. Philos. Trans. Roy. Soc. London Ser. A 157 (1866), 49-88. Reprinted in The scientific letters and papers of James Clerk Maxwell. Vol. II. 1862-1873, 26-78. Dover, New York, 1965.

[41] T. Nishida: Fluid dynamical limit of the nonlinear Boltzmann equation to the level of the compressible Euler equation. Comm. Math. Phys. 61 (1978), 119-148.

[42] L. Saint-Raymond: From the BGK model to the Navier-Stokes equations, Ann. Sci. Ecole. Norm. Sup. 36, 271-317 (2003) 